\title{
Understanding Neurosurgery Through Experimental and Computer Models
}

\author{
Ciro Randazzo MD \\ Thomas Jefferson University \\ Aditya S. Pandey MD \\ Thomas Jefferson University \\ Erol Veznedaroglu MD \\ Thomas Jefferson University
}

\section{Follow this and additional works at: https://jdc.jefferson.edu/jhnj \\ Part of the Neurology Commons \\ Let us know how access to this document benefits you}

\author{
Recommended Citation \\ Randazzo MD, Ciro; Pandey MD, Aditya S.; and Veznedaroglu MD, Erol (2008) "Understanding \\ Neurosurgery Through Experimental and Computer Models," JHN Journal: Vol. 4 : Iss. 1 , Article 6. \\ DOI: https://doi.org/10.29046/JHNJ.004.1.003 \\ Available at: https://jdc.jefferson.edu/jhnj/vol4/iss1/6
}

This Article is brought to you for free and open access by the Jefferson Digital Commons. The Jefferson Digital Commons is a service of Thomas Jefferson University's Center for Teaching and Learning (CTL). The Commons is a showcase for Jefferson books and journals, peer-reviewed scholarly publications, unique historical collections from the University archives, and teaching tools. The Jefferson Digital Commons allows researchers and interested readers anywhere in the world to learn about and keep up to date with Jefferson scholarship. This article has been accepted for inclusion in JHN Journal by an authorized administrator of the Jefferson Digital Commons. For more information, please contact: JeffersonDigitalCommons@jefferson.edu. 


\section{Understanding Neurosurgery Through Experimental and Computer Models}

\author{
Ciro Randazzo, MD, Aditya S. Pandey, MD, Erol Veznedaroglu, MD \\ Department of Neurological Surgery, Thomas Jefferson University, Philadelphia, Pennsylvania
}

\section{Introduction}

Endovascular neurosurgery is an evolving field, with the goal of treating neurological disease with minimal invasion of the body. The current approach is to deliver focused therapies via catheters traveling through the vascular tree. Refinement and advancement of these techniques requires not just new ideas, but new ideas that hold up when tested through the scientific method.

Before clinical trials can begin, ethics and law demand that the ideas are tested first under experimental models. One benefit of these pre-clinical tests is the early identification of side effects. Another is the opportunity to practice and polish surgical technique in advance of human surgery.

Historically, the best models of human systems have been similar systems in appropriate animals. Like human care, animal use is governed by strict laws and regulations. Violating these rules can not only result in criminal penalties, they can also make the research worthless: an animal poorly cared for may have physical symptoms that mask or confound the response to treatment.

As computing power has increased, mathematical models have become more popular. The advantages of computational models include relative costs and relative speed. No animals, drugs or equipment have to be purchased, just a computer and software. The disadvantage is that the computational model can only represent those aspects of the modeled system that are understood and quantifiable.

This article is an overview of how both animal and computational models have been used to approximate neurovascular conditions by researchers seeking to explore treatment options.

\section{Animal Models}

\section{Aneurysm}

\section{$\operatorname{Dog}$}

A common animal model for aneurysms is the canine, in which aneurysms are surgically constructed most often through the use of venous pouches. This model was first used in 1954 by German and Black ${ }^{1}$. Shin and Niimi later revised it ${ }^{2}$ to allow the creation of four kinds of aneurysm-bifurcation, side-wall, arterial stump and small branch, each with different hemodynamics-in one dog. The canine models have been consistently used due to their ease of handling and anesthesia, the similarity of the blood vessel size to that in humans, and the reproducibility of the studies. However, the canine has a tendency toward rapid lysis of clots, which limits its validity as a model for testing thrombosis of aneurysms after embolization ${ }^{3,4}$

\section{Rabbit}

A rabbit model of bifurcation aneurysms has been described by Spetzger and Reul. ${ }^{5}$ Although the rabbit has a similar blood clotting system to that of the human - an advantage missing in the dogthis model was limited due to high rates of pneumonia, post-operative neurologic complications, and peri- and post-operative mortality. There was also a high likelihood of inadvertent vessel occlusion seen on angiography after creation of the aneurysms. Only about half of the procedures yielded surgically created aneurysms which could be treated by coil embolization.

\section{Pig}

Venous pouch aneurysms have also been created on the common carotid artery in swine ${ }^{6}$ An advantage of the swine model is that spontaneous rupture of aneurysms, like those seen in humans, can occur'; experimental aneurysms in other animal models do not tend to rupture spontaneously. To construct the model, an anastomosis is created between the common carotid artery and the external jugluar vein. Retaining sutures are used to create a pouch in the proximal draining external jugular vein. After the animal heals for one to two weeks, an endovascular

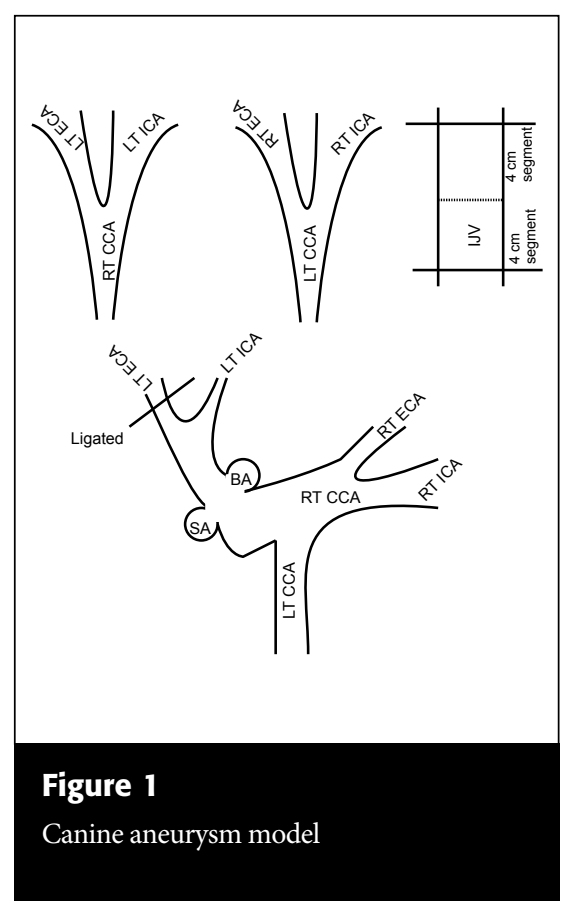

balloon can be placed angiographically to close the fistula, thus creating a fusiform vein pouch aneurysm which can subsequently be used for testing embolization devices.

Rat

Rats are popular in research for simple economic reasons: they are cheap to purchase and cheap to house. Many experimental studies require a comparison of treatments in several subjects; a large number of dogs or pigs would be prohibitively costly, and we have already seen that rabbits had a poor experimental yield. In a study of twenty rats, ten receiving Gugliemi detachable coils and ten receiving biodegradable polyglycolide coils $^{8}$, the aneurysms were created by permanent occlusion of the common carotid artery (CCA) just proximal to its bifurcation, creating an arteriotomy between the two ligatures and directly depositing segments of coils into the CCA. Although this model was useful to test the response of arteries to the deposited segments of coils, it was 
limited because the end-artery did not have flow or anatomical characteristics similar to aneurysms. Vein-pouch aneurysm models have also been described in rats. ${ }^{9,10}$

\section{Monkey}

Because monkeys are so closely related to humans, they would be considered the most accurate model for most systems. In an effort to more closely mimic the anatomy and hematologic clotting characteristics of the human, Tenjin and Ueda developed a primate aneurysm model. ${ }^{11}$ They used femoral vein pouches to create venous pouch aneurysms bilaterally on the carotid arteries of Japanese monkeys. They noted a success rate of $74 \%$ at two weeks in creating their aneurysms.

\section{Arteriovenous Malformations (AVMs)}

\section{Pig}

Pig vessels approximate the size of human vessels, their coagulation profile is similar to that in the human and they are fairly simple to anaesthetize. With these advantages, the pig model is the most commonly used and described model of cerebral AVMs. The swine, and other mammals including sheep, goat, ox and cat, provide blood supply to the brain via an arterial network of vessels, known as the rete mirabile, which is surrounded by the cavernous sinus at the base of the brain. Because the rete mirabile is a purely arterial network (as seen in other mammals including sheep, goat, ox and cat), a valid AVM model is created as an arterio-venous fistula formed by isolating and anastomosing the external jugular vein and common carotid artery on one side of the neck. ${ }^{3,4}$ The creation of this fistula with occlusion

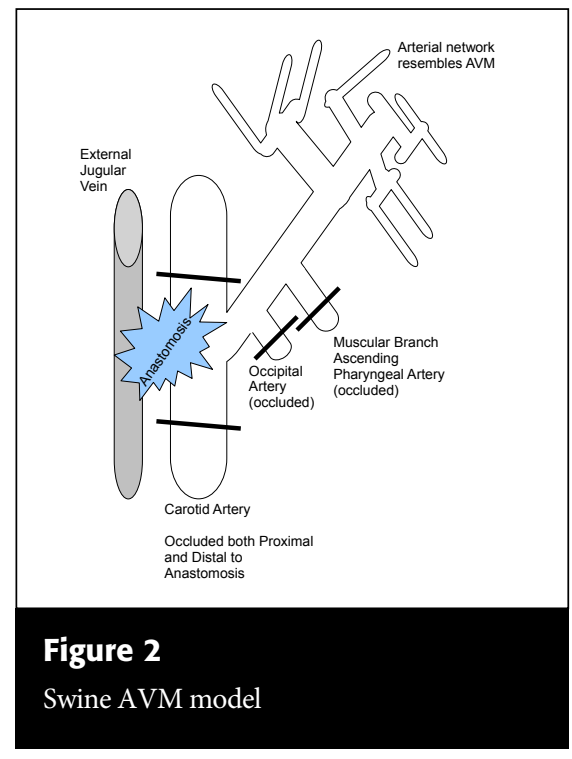

of carotid artery branches has been shown to increase flow and pressures across the rete ${ }^{12-14}$ and result in histologic changes to vessel walls very similar to those seen in human cerebral AVMs. ${ }^{15}$ This model has been used repeatedly for training in endovascular techniques, to evaluate nascent embolic materials and for histologic evaluations of radiosurgical induced changes. ${ }^{16,17}$

\section{Dog}

The only canine AVM model described is also the only completely intracerebral AVM model. Pietilä et al. ${ }^{18}$ created an arteriovenous fistula (AVF) by interposing a segment of superficial temporal artery between a main branch of the middle cerebral artery and the sagittal sinus. They concurrently implanted a muscle graft supplied by a branch of the fistula onto the ischemic area of the brain. Their intent was to have the ischemia serve as an angiogenic stimulus and thus mimic the angiogenesis that occurs in human AVMs. The authors found that many other aspects of this model mimicked human AVMs, including: thickening and fibrosis of the wall of the draining vein; angiogenesis surrounding the area of the created AVM with new vessels demonstrating pathological wall changes; gliotic brain tissue formation and areas of ischemia; hemorrhage and thrombus formation in the brain tissue and vessels surrounding the created nidus. The authors did not however, report any attempts at experimental treatments of this model.

\section{Rat}

In the rat, Bederson ${ }^{19}$ created an extracranial arteriovenous fistula with occlusion of the contralateral vein. This was first created in an attempt to determine if a dural AVM would form as a result of sinus thrombosis. This model has been further modified to evaluate cerebral hypoperfusion $^{20}$, the role of radiotherapy in the treatment of AVFs/AVMs ${ }^{21}$, and the role of sinus hypertension and thrombosis in the development of dural AVMs. ${ }^{22}$

\section{Vasospasm}

Cerebral vasospasm continues to be one of the major causes of morbidity and mortality following anuerysmal subarachnoid hemorrhage. Megyesi et al. published a review of fifty-seven in vivo animal models of cerebral vasospasm dating back to $1928 .^{23}$ These models employed rats, rabbits, cats, pigs, canines and primates. $72 \%$ used a technique in which blood was instilled around blood vessels to cause vasospasm and the remainder used a technique whereby they punctured or lacerated a vessel to allow blood to escape and thus induce vasospasm. Some of these models involve open cranial surgery to puncture the vessels, while others have implemented perforating the vessel from the luminal side by using an endovascular approach. They also describe extracranial models of vasospasm although they do point out that these models are controversial because their validity is questionable.

\section{Simulations}

\section{Aneurysm}

In a joint project between the departments of Neurosurgery and Aerospace Engineering, Kim et al. $^{24}$ used a computational fluid dynamics software package to assess the risk of aneurysm rupture when porous stents were used as the sole treatment. Such models are reliable to the point where they can predict angiograms. ${ }^{25} \mathrm{~A}$ model of a wide-necked basilar trunk aneurysm was constructed based on the patient's CT scans. The aneurysm was modeled untreated and treated with 1-3 stents from various manufacturers. The model predicted that these stents could suppress complex blood flow associated with ruptured aneurysms in another simulation study ${ }^{26}$, although the size of the effect depended on the specific geometry and on how well the stents were deployed and that a single stent would probablybeinsufficient to ensure a good outcome.

\section{Arteriovenous Malformations (AVMs)}

Hademenos and Massoud created a mathematical model based on electrical networks. ${ }^{27,} 28$ This model was designed to allow an analysis of changes in AVM hemodynamic and biophysical characteristics, and individual parameters could be manipulated to determine changes in flow characteristic and likelihood of rupture or hemorrhage after various treatments.

Kerber and $\mathrm{Hecht}^{29}$ implemented on a personal computer (with an Intel 286 microprocessor!) a mathematical model which represented the AVM as a collection of 1000 channels, each of which must be embolized to "cure" the simulated AVM. They later ${ }^{30}$ constructed a physical model from a sponge with waxed tubes representing inflow (arterial feeders) and outflow tracts (draining veins). These models were intended to help guide the development of new neurointerventional techniques. The physical model made it further possible to test liquid embolic materials using microcatheters, and to teach endovascular embolization techniques in a no-risk situation. 


\section{Applications in Training}

In an ongoing effort to promote patient safety and enhance skills training, recent advances in technology have contributed to the development of non-animal simulation technology. These simulators enhance learning fundamental skills in procedures such as basic suturing, laparscopic surgery and endovascular treatments. As the technologies improve, the fidelity of these models has improved the reliability of these systems. For example, Wong et al. ${ }^{31}$ describe a $3 \mathrm{D}$ virtual reality system integrated with simulated manipulators for training in craniotomies and aneurysm clipping. A variety of clips and patient aneurysms were scanned into the simulator database so the user could learn how and why to combine different clips with different surgical approaches. Another group ${ }^{32,33}$ used the same system to train clinicians in minimally invasive surgical techniques.

The development of haptic technologies, which provide touch and pressure feedback, have significantly improved the tactile experience of simulators. ${ }^{34,35}$ Currently in use at the Jefferson Hospital for Neuroscience (JHN) is the Procedicus VIST ${ }^{\mathrm{ma}}$ simulator. This is a virtual reality simulator system that uses haptic feedback and metric assessment. ${ }^{36,37}$ It can be used to acquire and hone basic and complex neuroendovascular skills. The system utilizes a mannequin and two computer screens along with actual endovascular equipment. The computer allows the user to choose from a variety of different programs representing different pathologies, e.g. saccular aneurysms, fusiform aneurysms, carotid stenosis.

The simulator at JHN has been used by neurointerventional technologists, residents and fellows to develop proficiency in various procedures. This leads to increased benefits for practitioners, as they have improved skills and confidence when performing procedures on patients. The simulator has also improved patient safety as simulators have been shown to improve performance in endovascular procedures. $^{38}$

\section{Conclusion}

The new era of evidence-based management of disease will continue to demand increased justification from physicians for the treatments they use. At the same time, ethical constraints limit the early testing of new technologies on patients. Animal models, when designed, validated and used appropriately, can be an invaluable resource in the development of lifesaving technologies and techniques.
One reason to model a biological system is that construction of the model proves enough understanding to reproduce the system. It is presumably that same understanding that makes neurosurgical repair of the patient possible. A potential advantage of computational models is the opportunity to perform many virtual experiments in less time than it would take to perform one real experiment, but this depends on the power of the computer relative to the complexity of the model. An extension of this is using a computer to design optimized surgical plans and project the likely outcomes for patients in advance of surgery. The potential of computer models is restricted by the bottom limit of our ability to observe physiological processes and represent them on computer: as yet, we may not even have approached that limit.

\section{References}

1. German, W.J. and S.P. Black, Experimental production of carotid aneurysms. N Engl J Med, 1954. 250(3): p. 104-6.

2. Shin, Y.S., et al., Creation of four experimental aneurysms with different hemodynamics in one dog. AJNR Am J Neuroradiol, 2005. 26(7): p. 1764-7.

3. Massoud, T.F., et al., Experimental saccular aneurysms. I. Review of surgically-constructed models and their laboratory applications. Neuroradiology, 1994. 36(7): p. 537-46.

4. Massoud, T.F., et al., Experimental models of bifurcation and terminal aneurysms: construction techniques in swine AJNR Am J Neuroradiol, 1994. 15(5): p. 938-44.

5. Spetzger, U., et al., Microsurgically produced bifurcation aneurysms in a rabbit model for endovascular coil embolization. J Neurosurg, 1996. 85(3): p. 488-95.

6. Guglielmi, G., et al., Experimental saccular aneurysms. II. A new model in swine. Neuroradiology, 1994. 36(7): p. $547-50$

7. Byrne, J.V. and N. Hubbard, A novel two-stage technique for construction of experimental aneurysms. AJNR Am J Neuroradiol, 2004. 25(2): p. 319-21.

8. Abrahams, J.M., et al., Biodegradable polyglycolide endovascular coils promote wall thickening and drug delivery in a rat aneurysm model. Neurosurgery, 2001. 49(5): p. 118793; discussion 1193-5.

9. Kirse, D.J., et al., Construction of a vein-pouch aneurysm at a surgically created carotid bifurcation in the rat. Microsurgery, 1996. 17(12): p. 681-9.

10. Young, P.H. and M.G. Yasargil, Experimental carotid artery aneurysms in rats: a new model for microsurgical practice. J Microsurg, 1982. 3(3): p. 135-46.

11. Tenjin, H., et al., Effect of Guglielmi detachable coils on experimental carotid artery aneurysms in primates. Stroke, 1995. 26(11): p. 2075-80.

12. Siekmann, R., et al., Modification of a previously described arteriovenous malformation model in the swine: endovascular and combined surgical/endovascular construction and hemodynamics. AJNR Am J Neuroradiol, 2000. 21(9): p. 1722-5.

13. Murayama, Y., T.F. Massoud, and F. Vinuela, Transvenous hemodynamic assessment of experimental arteriovenous malformations. Doppler guidewire monitoring of embolotherapy in a swine model. Stroke, 1996. 27(8): p 1365-72.

14. Murayama, Y., T.F. Massoud, and F. Vinuela, Hemodynamic changes in arterial feeders and draining veins during embolotherapy of arteriovenous malformations: an experimental study in a swine model. Neurosurgery, 1998. 43(1): p. 96-104; discussion 104-6.

15. Massoud, T.F., Experimental arteriovenous malformations modeling in laboratory sheep versus swine. AJNR Am J Neuroradiol, 2000. 21(5): p. 985-90.
16. Massoud, T.F., et al., Laboratory simulations and training in endovascular embolotherapy with a swine arteriovenous malformation model. AJNR Am J Neuroradiol, 1996. 17(2): p. $271-9$.

17. Jahan, R., et al., An arteriovenous malformation model for stereotactic radiosurgery research. Neurosurgery, 2007. 61(1): p. 152-9; discussion 159

18. Pietila, T.A., et al., Animal model for cerebral arteriovenous malformation. Acta Neurochir (Wien), 2000. 142(11): p. 1231-40

19. Bederson, J.B., et al., Intracranial venous hypertension and the effects of venous outflow obstruction in a rat model of arteriovenous fistula. Neurosurgery, 1991. 29(3): p. 341-50

20. Hai, J., et al., A new rat model of chronic cerebral hypoperfusion associated with arteriovenous malformations. J Neurosurg, 2002. 97(5): p. 1198-202.

21. Altschuler, E., et al., Radiobiologic models for radiosurgery Neurosurg Clin N Am, 1992. 3(1): p. 61-77.

22. Herman, J.M., et al., Genesis of a dural arteriovenous malformation in a rat model. J Neurosurg, 1995. 83(3): p. 539-45.

23. Megyesi, J.F., et al., In vivo animal models of cerebral vasospasm: a review. Neurosurgery, 2000. 46(2): p. 448-60; discussion 460-1.

24. Kim, M., et al., Quantification of hemodynamic changes induced by virtual placement of multiple stents across a wide-necked basilar trunk aneurysm. Neurosurgery, 2007. 61(6): p. 1305-12; discussion 1312-3.

25. Cebral, J.R., R.S. Pergolizzi, Jr., and C.M. Putman, Computational fluid dynamics modeling of intracranial aneurysms: qualitative comparison with cerebral angiography. Acad Radiol, 2007. 14(7): p. 804-13.

26. Cebral, J.R., et al., Characterization of cerebral aneurysms for assessing risk of rupture by using patient-specific computational hemodynamics models. AJNR Am J Neuroradiol, 2005. 26(10): p. 2550-9.

27. Hademenos, G.J. and T.F. Massoud, An electrical network model of intracranial arteriovenous malformations: analysis of variations in hemodynamic and biophysical parameters. Neurol Res, 1996. 18(6): p. 575-89.

28. Hademenos, G.J., T.F. Massoud, and F. Vinuela, A biomathematical model of intracranial arteriovenous malformations based on electrical network analysis: theory and hemodynamics. Neurosurgery, 1996. 38(5): p. 1005-14; discussion 1014-5.

29. Hecht, S.T., J.A. Horton, and C.W. Kerber, Hemodynamic of the central nervous system arteriovenous malformation nidus during particulate embolization. A computer model. Neuroradiology, 1991. 33(1): p. 62-4.

30. Kerber, C.W., S.T. Hecht, and K. Knox, Arteriovenous malformation model for training and research. AJNR Am J Neuroradiol, 1997. 18(7): p. 1229-32.

31. Wong, G.K., et al., Craniotomy and clipping of intracranial aneurysm in a stereoscopic virtual reality environment. Neurosurgery, 2007. 61(3): p. 564-8; discussion 568-9.

32. Kockro, R.A., et al., A collaborative virtual reality environment for neurosurgical planning and training. Neurosurgery, 2007. 61(5 Suppl 2): p. 379-91; discussion 391.

33. Stadie, A.T., et al., Virtual reality system for planning minimally invasive neurosurgery. Technical note. J Neurosurg, 2008. 108(2): p. 382-94.

34. Scalese, R.J. and S.B. Issenberg, Effective use of simulation for the teaching and acquisition of veterinary professional and clinical skills. J Vet Med Educ, 2005. 32(4): p. 461-7.

35. Scalese, R.J., V.T. Obeso, and S.B. Issenberg, Simulation technology for skills training and competency assessment in medical education. J Gen Intern Med, 2008. 23 Suppl 1: p. 46-9.

36. Neequaye, S.K., et al., Identification of skills common to renal and iliac endovascular procedures performed on a virtual reality simulator. Eur J Vasc Endovasc Surg, 2007. 33(5): p. 525-32.

37. Neequaye, S.K., et al., Endovascular skills training and assessment. J Vasc Surg, 2007. 46(5): p. 1055-64.

38. Aggarwal, R., et al., Virtual reality simulation training can improve inexperienced surgeons' endovascular skills. Eur Vasc Endovasc Surg, 2006. 31(6): p. 588-93. 